\title{
On the dynamics of the nonlinear difference equation $x_{n+1}=\alpha+\beta x_{n-1}+x_{n-1} / x_{n}$
}

\author{
Aycan Aksoy, Mehmet Turan* \\ Atilim University, Department of Mathematics, Incek 06836, Ankara, Turkey \\ *Corresponding author, e-mail: mehmet.turan@atilim.edu.tr
}

Received 18 Feb 2015

Accepted 12 Oct 2015

ABSTRACT: The boundedness and semi-cycle analysis of positive solutions, existence of period-2 solutions, and local and global asymptotic stability of the recursive sequence $x_{n+1}=\alpha+\beta x_{n-1}+x_{n-1} / x_{n}, n=0,1, \ldots$ are investigated where $\alpha \in[0, \infty), \beta \in[0,1)$ and the initial conditions $x_{-1}$ and $x_{0}$ are arbitrary positive real numbers. The paper concludes with some numerical examples to illustrate the theoretical results.

KEYWORDS: recursion relation, stability, boundedness

MSC2010: 39A10 39A22 39A23 39A30

\section{INTRODUCTION}

Difference equations can be used to model and analyse many real-world processes where the current state is evaluated in terms of some previous states. Because of their wide range of applications ${ }^{1-4}$ many researchers have studied these systems ${ }^{5-8}$.

The aim of this paper is to examine the boundedness character and the semi-cycle analysis of the positive solutions, the periodic nature, and the stability of the difference equation

$$
x_{n+1}=\alpha+\beta x_{n-1}+\frac{x_{n-1}}{x_{n}}, \quad n=0,1, \ldots,
$$

where $\alpha \in[0, \infty), \beta \in[0,1)$ and the initial conditions $x_{-1}$ and $x_{0}$ are arbitrary positive real numbers. Equation (1) with $\beta=0$ becomes

$$
x_{n+1}=\alpha+\frac{x_{n-1}}{x_{n}}, \quad n=0,1, \ldots,
$$

which has been dealt with by many authors. Also, the recursive sequence (2) for negative values of $\alpha$ has been examined in Refs. 9, 10, and for nonnegative values of $\alpha$ has been studied in Ref. 11 . Some of the types of behaviour that are studied in this paper have been investigated in Refs. 12, 13 for $x_{n+1}=\alpha+x_{n-k} / x_{n}$ for $k \in \mathbb{Z}^{+}$.

Equation (1) can be written as

$$
x_{n+1}=f\left(x_{n}, x_{n-1}\right), \quad n=0,1, \ldots,
$$

where $f(x, y)=\alpha+\beta y+y / x$. Since $f:(0, \infty) \times$ $(0, \infty) \rightarrow(0, \infty)$ is a continuously differentiable function, (3) and hence (1) have a unique solution $\left\{x_{n}\right\}_{n=-1}^{\infty}$ for all initial conditions $x_{-1}, x_{0} \in(0, \infty)$.

\section{PRELIMINARIES}

The definitions provided in this section can be found in many books ${ }^{2,7}$ and papers (see Refs. 9,11 and the references therein), and the preliminary results are either given in these references or can be derived as a simple consequence of those obtained in there.

A point $\bar{x} \in(0, \infty)$ is said to be a fixed point or an equilibrium solution of (3) if $f(\bar{x}, \bar{x})=\bar{x}$. Clearly, the only fixed point of (1) is

$$
\bar{x}=(1+\alpha) /(1-\beta) .
$$

Let $\left\{x_{n}\right\}_{n=-1}^{\infty}$ be a positive solution of (1). A positive semi-cycle of $\left\{x_{n}\right\}_{n=-1}^{\infty}$ consists of a string of terms $\left\{x_{l}, x_{l+1}, \ldots, x_{m}\right\}$, all greater than or equal to $\bar{x}$, with $l \geqslant-1$ and $m \leqslant \infty$ and such that

$$
\text { either } l=-1, \quad \text { or } \quad l>-1 \text { and } x_{l-1}<\bar{x}
$$

and

$$
\text { either } m=\infty \text {, or } m<\infty \text { and } x_{m+1}<\bar{x} \text {. }
$$

A negative semi-cycle of $\left\{x_{n}\right\}_{n=-1}^{\infty}$ consists of a string of terms $\left\{x_{l}, x_{l+1}, \ldots, x_{m}\right\}$, all less than $\bar{x}$, with $l \geqslant$ -1 and $m \leqslant \infty$ and such that

$$
\text { either } l=-1, \quad \text { or } \quad l>-1 \text { and } x_{l-1} \geqslant \bar{x}
$$

and

either $m=\infty$, or $\quad m<\infty$ and $x_{m+1} \geqslant \bar{x}$.

A solution $\left\{x_{n}\right\}_{n=-1}^{\infty}$ of (1) is nonoscillatory if there exists $N \geqslant-1$ such that either

$$
x_{n}>\bar{x} \text { for all } n \geqslant N
$$


or

$$
x_{n}<\bar{x} \text { for all } n \geqslant N .
$$

A solution $\left\{x_{n}\right\}_{n=-1}^{\infty}$ is called oscillatory if it is not nonoscillatory.

A fixed point $\bar{x}$ of (1) is said to be locally stable if there exists an interval $I \subset(0, \infty)$ such that to any $\epsilon>0$ there corresponds a $\delta=\delta(\epsilon)>0$ with the property that $x_{-1}, x_{0} \in I$ and $\left|x_{-1}-\bar{x}\right|+$ $\left|x_{0}-\bar{x}\right|<\delta$ implies $\left|x_{n}-\bar{x}\right|<\epsilon$ for all $n \geqslant-1$; locally asymptotically stable if it is locally stable and there exists $\gamma>0$ such that $x_{-1}, x_{0} \in I$ and $\left|x_{-1}-\bar{x}\right|+\left|x_{0}-\bar{x}\right|<\gamma$ implies $\lim _{n \rightarrow \infty} x_{n}=\bar{x}$; a global attractor if $\lim _{n \rightarrow \infty} x_{n}=\bar{x}$ for any $x_{-1}, x_{0} \in I$; globally asymptotically stable if it is locally asymptotically stable and a global attractor; and unstable if it is not locally stable.

In general, the local stability analysis of the fixed point of a nonlinear equation is carried out by means of linearization about the fixed point. Setting $A=(\partial f / \partial x)(\bar{x}, \bar{x})$ and $B=(\partial f / \partial y)(\bar{x}, \bar{x})$, one can write the so-called linearized equation of (3) about $\bar{x}$ as

$$
y_{n+1}=A y_{n}+B y_{n-1}, \quad n=0,1, \ldots,
$$

whose characteristic equation is

$$
\lambda^{2}-A \lambda-B=0 .
$$

Let $\lambda_{1}$ and $\lambda_{2}$ denote the roots of (5). Then the following theorem holds ${ }^{7}$.

Theorem 1 (Linear stability)

(i) If $\left|\lambda_{1}\right|<1$ and $\left|\lambda_{2}\right|<1$, then the fixed point $\bar{x}$ of (1) is locally asymptotically stable.

(ii) If $\left|\lambda_{1}\right|>1$ or $\left|\lambda_{2}\right|>1$, then the fixed point $\bar{x}$ of (1) is unstable.

(iii) A necessary and sufficient condition for both roots of (5) to lie in the open unit disk $|\lambda|<1$ is

$$
|A|<1-B<2 .
$$

(iv) A necessary and sufficient condition for at least one root of (5) to have $|\lambda|>1$ is

$$
|B|>1 \text { and }|A|<|1-B|
$$

or

$$
A^{2}+4 B>0 \text { and }|A|>|1-B| .
$$

A solution $\left\{x_{n}\right\}_{n=-1}^{\infty}$ of (1) is said to be periodic with period $p$ if $x_{n+p}=x_{n}$ for all $n \geqslant-1$. The smallest positive integer $p$ is called the prime period of $\left\{x_{n}\right\}_{n=-1}^{\infty}$.
Lemma 1 Let $\left\{x_{n}\right\}_{n=-1}^{\infty}$ be a solution of (1), and let $L>\alpha /(1-\beta)$. Then

(i) $\lim _{n \rightarrow \infty} x_{2 n}=L \Leftrightarrow \lim _{n \rightarrow \infty} x_{2 n+1}=L /[(1-$ $\beta) L-\alpha]$

(ii) $\lim _{n \rightarrow \infty} x_{2 n+1}=L \Leftrightarrow \lim _{n \rightarrow \infty} x_{2 n}=L /[(1-$ $\beta) L-\alpha]$.

Proof: Taking the limit of (1) as $n \rightarrow \infty$ yields the required result.

Lemma 2 Let $\left\{x_{n}\right\}_{n=-1}^{\infty}$ be a positive solution of (1). Then the following statements are true for all $n$.

(i) $x_{n+1}<x_{n-1} \Leftrightarrow x_{n-1}+\alpha x_{n}+(\beta-1) x_{n-1} x_{n}<0$.

(ii) $x_{n+1}=x_{n-1} \Leftrightarrow x_{n-1}+\alpha x_{n}+(\beta-1) x_{n-1} x_{n}=0$.

(iii) $x_{n+1}>x_{n-1} \Leftrightarrow x_{n-1}+\alpha x_{n}+(\beta-1) x_{n-1} x_{n}>0$.

Proof: The lemma follows immediately from the fact that

$$
\begin{aligned}
x_{n+1}-x_{n-1} & =\alpha+\beta x_{n-1}+\frac{x_{n-1}}{x_{n}}-x_{n-1} \\
& =\frac{x_{n-1}+\alpha x_{n}+(\beta-1) x_{n-1} x_{n}}{x_{n}} .
\end{aligned}
$$

Corollary 1 Let $\left\{x_{n}\right\}_{n=-1}^{\infty}$ be a positive solution (1), and $\alpha=1$. Then

(i) If $x_{-1}<x_{1}$, then $x_{-1}<x_{1}<x_{3}<\cdots$ and $x_{0}<$ $x_{2}<x_{4}<\cdots$

(ii) if $x_{-1}=x_{1}$, then $x_{-1}=x_{1}=x_{3}=\cdots$ and $x_{0}=$ $x_{2}=x_{4}=\cdots$;

(iii) if $x_{-1}>x_{1}$, then $x_{-1}>x_{1}>x_{3}>\cdots$ and $x_{0}>$ $x_{2}>x_{4}>\cdots$.

Proof: Observe that, for $n \geqslant 0$,

$$
\begin{aligned}
& x_{n}+x_{n+1}+(\beta-1) x_{n} x_{n+1}= \\
& \frac{\left(1+\beta x_{n}\right)\left(x_{n-1}+x_{n}+(\beta-1) x_{n-1} x_{n}\right)}{x_{n}}
\end{aligned}
$$

and, hence by Lemma 2 with $\alpha=1$ one has the required results.

Theorem 2 of Ref. 10 states that if $\alpha_{n}$ is a period2 sequence, $f$ and $g$ are non-decreasing continuous functions which map the interval $(0, \infty)$ into itself, and $\left\{x_{n}\right\}$ is a positive solution of

$$
x_{n}=\alpha_{n}+\frac{f\left(x_{n-2}\right)}{g\left(x_{n-1}\right)},
$$

then the sequences $\left\{x_{2 n}\right\}$ and $\left\{x_{2 n+1}\right\}$ are eventually monotonic.

Taking $\alpha_{n}=\alpha, f(x)=x$ and $g(x)=x /(\beta x+1)$, in Theorem 2 of Ref. 10, the following result can be deduced. 
Lemma 3 Let $\alpha \geqslant 0,0 \leqslant \beta<1$, and $\left\{x_{n}\right\}_{n=-1}^{\infty}$ be a positive solution of (1). Then $\left\{x_{2 n}\right\}_{n=0}^{\infty}$ and $\left\{x_{2 n-1}\right\}_{n=0}^{\infty}$ are eventually monotonic.

\section{MAIN RESULTS}

\section{Boundedness}

In this part, the boundedness of positive solutions of (1) is addressed. For this purpose, firstly the following lemma which will be important to prove the existence of an unbounded solution is given.

Lemma 4 Let $\alpha \geqslant 0,0 \leqslant \beta<1$, and $\left\{x_{n}\right\}_{n=-1}^{\infty}$ be a positive solution of (1). Then at least one of the subsequences $\left\{x_{2 n}\right\}_{n=0}^{\infty}$ and $\left\{x_{2 n-1}\right\}_{n=0}^{\infty}$ is bounded. Also,

(i) $\lim _{n \rightarrow \infty} x_{2 n}=\infty \Leftrightarrow \lim _{n \rightarrow \infty} x_{2 n-1}=\alpha /(1-\beta)$.

(ii) $\lim _{n \rightarrow \infty} x_{2 n-1}=\infty \Leftrightarrow \lim _{n \rightarrow \infty} x_{2 n}=\alpha /(1-$ $\beta)$.

Proof: Suppose that $\left\{x_{n}\right\}_{n=-1}^{\infty}$ is a positive solution of (1) such that both $\left\{x_{2 n}\right\}_{n=0}^{\infty}$ and $\left\{x_{2 n-1}\right\}_{n=0}^{\infty}$ are unbounded. Using Lemma 3 , it is easy to see that $\lim _{n \rightarrow \infty} x_{2 n}=\infty$ and $\lim _{n \rightarrow \infty} x_{2 n+1}=\infty$. Then

$$
\lim _{n \rightarrow \infty} \frac{x_{2 n+1}}{x_{2 n-1}}=\lim _{n \rightarrow \infty}\left(\frac{\alpha}{x_{2 n-1}}+\beta+\frac{1}{x_{2 n}}\right)=\beta .
$$

Now, for $\epsilon=(1-\beta) / 2$, there exists $N \in \mathbb{N}$ such that

$$
\left|\frac{x_{2 n+1}}{x_{2 n-1}}-\beta\right|<\frac{1-\beta}{2} \text { for all } n>N,
$$

which gives us $x_{2 n+1}<\frac{1}{2}(1+\beta) x_{2 n-1}$ for all $n>N$. Using this inequality repeatedly, one obtains

$$
x_{2 n+1}<\left(\frac{1+\beta}{2}\right)^{n-N} x_{2 N+1} \text { for all } n>N .
$$

Since $(1+\beta) / 2<1$, the above estimate leads to $\lim _{n \rightarrow \infty} x_{2 n+1}=0$, which is a contradiction. Additionally, one can show that if one of the subsequences $\left\{x_{2 n}\right\}_{n=0}^{\infty}$ and $\left\{x_{2 n-1}\right\}_{n=0}^{\infty}$ is unbounded, then the other one converges to $\alpha /(1-\beta)$.

In the next theorem, it is shown that there exist positive solutions of (1) which are unbounded.

Theorem 2 Let $0 \leqslant \alpha<1,0 \leqslant \beta<1$, and $\left\{x_{n}\right\}_{n=-1}^{\infty}$ be a solution of (1) satisfying $0<x_{-1}<1 /(1-\beta)$ and $x_{0}>1 /[(1-\alpha)(1-\beta)]$. Then

$$
\lim _{n \rightarrow \infty} x_{2 n}=\infty \text { and } \lim _{n \rightarrow \infty} x_{2 n+1}=\frac{\alpha}{1-\beta} .
$$

Proof: Since $0 \leqslant \alpha<1$, it is clear that $1 /(1-\alpha) \geqslant$ $\alpha+1$. Hence $x_{0}>\bar{x}$. Also,

$$
x_{1}=\alpha+\beta x_{-1}+\frac{x_{-1}}{x_{0}}<\frac{1}{1-\beta}
$$

and

$$
x_{1}=\alpha+\beta x_{-1}+\frac{x_{-1}}{x_{0}}>\alpha .
$$

That is, $\alpha<x_{1}<1 /(1-\beta)$. On the other hand,

$$
\begin{aligned}
& x_{2}=\alpha+\beta x_{0}+\frac{x_{0}}{x_{1}}=\alpha+\left(\beta+\frac{1}{x_{1}}\right) x_{0}>\alpha+x_{0}, \\
& x_{3}=\alpha+\beta x_{1}+\frac{x_{1}}{x_{2}}<\alpha+\beta x_{1}+\frac{x_{1}}{x_{0}}<\frac{1}{1-\beta} .
\end{aligned}
$$

By induction, one can show that

$$
\begin{aligned}
x_{2 n}>n \alpha+x_{0} \quad \text { and } \quad x_{2 n-1} & \in\left(\alpha, \frac{1}{1-\beta}\right) \\
& \text { for all } n \geqslant 1 .
\end{aligned}
$$

Hence if $\alpha \neq 0$, then $\lim _{n \rightarrow \infty} x_{2 n}=\infty$ and, hence by Lemma $4, \lim _{n \rightarrow \infty} x_{2 n+1}=\alpha /(1-\beta)$ as claimed.

For $\alpha=0$ one has

$$
x_{2 n+2}-x_{2 n}=\left(\beta-1+\frac{1}{x_{2 n+1}}\right) x_{2 n}>0
$$

and

$$
x_{2 n+1}-x_{2 n-1}=\left(\beta-1+\frac{1}{x_{2 n}}\right) x_{2 n-1}<0
$$

which means that $\left\{x_{2 n}\right\}$ is strictly increasing and $\left\{x_{2 n+1}\right\}$ is strictly decreasing. If $\lim _{n \rightarrow \infty} x_{2 n}=L<$ $\infty$, then by Lemma 1 one obtains $\lim _{n \rightarrow \infty} x_{2 n+1}=$ $1 /(1-\beta)$. Taking the limit as $n \rightarrow \infty$ on both sides of $x_{2 n+1}=\left(\beta+1 / x_{2 n}\right) x_{2 n-1}$ yields $L=1 /(1-\beta)$, which is not possible since $\left\{x_{2 n}\right\}$ is increasing and $x_{0} \geqslant \bar{x}$. Hence $\lim _{n \rightarrow \infty} x_{2 n}=\infty$ and, by Lemma 4, $\lim _{n \rightarrow \infty} x_{2 n+1}=0$ as required.

\section{Periodicity and semi-cycle analysis}

In this part, the period-2 solutions of (1) are considered. Also, the semi-cycle analysis of positive solutions is performed and the convergence of any positive solution to the fixed point or a period-2 solution of (1) is dealt with alongside this.

Lemma 5 Equation (1) has period-2 solutions if and only if $\alpha=1$. Moreover, when $\alpha=1,\left\{x_{n}\right\}_{n=-1}^{\infty}$ is period-2 if and only if $x_{-1} \neq 2 /(1-\beta), x_{-1} \neq 1 /(1-$ $\beta$ ) and $x_{0}=x_{-1} /\left(x_{-1}(1-\beta)-1\right)$.

Proof: Suppose that (1) has a period-2 solution 
where $x \neq y$. Then

$$
\begin{aligned}
& x=\alpha+\beta x+\frac{x}{y}, \\
& y=\alpha+\beta y+\frac{y}{x} .
\end{aligned}
$$

Subtraction of the latter equation from the former one yields $y=x /[x(1-\beta)-1]$. Plugging this into (8a) gives $\alpha=1$. Notice that $x=2 /(1-\beta)$ results in $y=2 /(1-\beta)$, which contradicts the assumption that $x \neq y$.

Conversely, assume that $\alpha=1$. Let $x_{-1} \neq 2 /(1-$ $\beta), x_{-1} \neq 1 /(1-\beta)$ and $x_{0}=x_{-1} /\left(x_{-1}(1-\beta)-1\right)$. From (1), the following can be deduced:

$$
\begin{aligned}
& x_{1}=1+\beta x_{-1}+\frac{x_{-1}}{x_{0}}=x_{-1} \\
& x_{2}=1+\beta x_{0}+\frac{x_{0}}{x_{1}}=1+\beta x_{0}+\frac{x_{0}}{x_{-1}}=x_{0} .
\end{aligned}
$$

By induction, it is now easy to see that $\left\{x_{n}\right\}_{n=-1}^{\infty}$ is a period-2 solution.

Lemma 6 Let $\left\{x_{n}\right\}_{n=-1}^{\infty}$ be a positive solution of (1) which consists of a single semi-cycle. Then $\left\{x_{n}\right\}_{n=-1}^{\infty}$ converges to $\bar{x}=(1+\alpha) /(1-\beta)$.

Proof: Suppose that $\left\{x_{n}\right\}_{n=-1}^{\infty}$ is a positive solution of (1) which is a negative semi-cycle. Then, using $1-\beta=(1+\alpha) / \bar{x}$ and $0<x_{n}<\bar{x}$, one obtains

$$
\begin{aligned}
x_{2 n+2}-x_{2 n} & =\alpha+\left(\beta-1+\frac{1}{x_{2 n+1}}\right) x_{2 n} \\
& >\alpha\left(1-\frac{x_{2 n}}{\bar{x}}\right) \geqslant 0
\end{aligned}
$$

and

$$
\begin{aligned}
x_{2 n+1}-x_{2 n-1} & =\alpha+\left(\beta-1+\frac{1}{x_{2 n}}\right) x_{2 n-1} \\
& >\alpha\left(1-\frac{x_{2 n-1}}{\bar{x}}\right) \geqslant 0,
\end{aligned}
$$

implying that the subsequences $\left\{x_{2 n+1}\right\}_{n=-1}^{\infty}$ and $\left\{x_{2 n}\right\}_{n=0}^{\infty}$ are both strictly increasing. Hence the limits $\lim _{n \rightarrow \infty} x_{2 n+1}=L_{1}$ and $\lim _{n \rightarrow \infty} x_{2 n}=L_{2}$ exist. Also, $L_{1}, L_{2} \in(0, \bar{x}]$. Since $L_{1}=\alpha+\beta L_{1}+L_{1} / L_{2}$, one has

$$
\frac{\alpha}{L_{1}}+\frac{1}{L_{2}}=1-\beta .
$$

Now, if $L_{1}<\bar{x}$ or $L_{2}<\bar{x}$, then $\alpha / L_{1}+1 / L_{2}>$ $(\alpha+1) / \bar{x}=1-\beta$, which contradicts (9). Hence $L_{1}=L_{2}=\bar{x}$ and, hence, $\left\{x_{n}\right\}_{n=-1}^{\infty}$ converges to $\bar{x}$, as claimed.

The case when $\left\{x_{n}\right\}_{n=-1}^{\infty}$ is a positive semi-cycle can be handled in a similar way.
Lemma 7 Let $\left\{x_{n}\right\}_{n=-1}^{\infty}$ be a positive solution of (1) which consists of at least two semi-cycles. Then $\left\{x_{n}\right\}_{n=-1}^{\infty}$ is oscillatory. Moreover, with the possible exception of the first semi-cycle, every semi-cycle has length 1. Aside from that, for any $\varepsilon>0$, except possibly for finitely many terms, every term of $\left\{x_{n}\right\}_{n=-1}^{\infty}$ is strictly greater than $\alpha /(1-\beta)-\varepsilon$.

Proof: Suppose that $\left\{x_{n}\right\}_{n=-1}^{\infty}$ is a positive solution which consists of at least two semi-cycles. Then there exists $m \geqslant-1$ such that $x_{m}<\bar{x} \leqslant x_{m+1}$ or $x_{m+1}<\bar{x} \leqslant x_{m}$. Only the former case will be considered since the latter can be treated similarly. Now,

$$
x_{m+2}=\alpha+\beta x_{m}+\frac{x_{m}}{x_{m+1}}<\alpha+\beta \bar{x}+1=\bar{x}
$$

and

$$
x_{m+3}=\alpha+\beta x_{m+1}+\frac{x_{m+1}}{x_{m+2}}>\alpha+\beta \bar{x}+1=\bar{x} .
$$

Again by induction, it can be shown that

$$
\alpha<x_{m+2 k}<\bar{x} \leqslant x_{m+2 k+1} \text { for } k \geqslant 0 .
$$

That is, every semi-cycle, except possibly for the first one, say $\left\{x_{-1}, \ldots, x_{m}\right\}$, has length 1 , and the solution $\left\{x_{n}\right\}_{n=-1}^{\infty}$ is oscillatory.

Additionally, by Lemma 3, it is clear that the subsequences $\left\{x_{m+2 k}\right\}_{k=0}^{\infty}$ and $\left\{x_{m+2 k+1}\right\}_{k=0}^{\infty}$ are eventually monotonic. Hence, $x_{m+2 k} \rightarrow L_{1}$ as $k \rightarrow \infty$, where $\alpha \leqslant L_{1} \leqslant \bar{x}$. In the case when $\left\{x_{m+2 k+1}\right\}_{k=0}^{\infty}$ is not bounded from above, one has $x_{m+2 k+1} \rightarrow \infty$ as $k \rightarrow \infty$ which, by Lemma 4 , implies that $L_{1}=$ $\alpha /(1-\beta)$. On the other hand, if $\left\{x_{m+2 k+1}\right\}_{k=0}^{\infty}$ is bounded from above, then it has a finite limit, say $L_{2}$. Clearly,

$$
\frac{\alpha}{L_{1}}+\frac{1}{L_{2}}=1-\beta=\frac{1}{L_{1}}+\frac{\alpha}{L_{2}},
$$

and $L_{1}>\alpha /(1-\beta)$ since, otherwise,

$$
1-\beta=\frac{\alpha}{L_{1}}+\frac{1}{L_{2}} \geqslant 1-\beta+\frac{1}{L_{2}}
$$

implies that $L_{2} \leqslant 0$, which is an obvious contradiction. Thus in all cases, $L_{1} \geqslant \alpha /(1-\beta)$. Using this together with (10), one obtains the final result of Lemma 7.

Theorem 3 Let $\alpha=1,0 \leqslant \beta<1$, and $\left\{x_{n}\right\}_{n=-1}^{\infty}$ be a positive solution of (1). Then the following statements hold: 
(i) if $\left\{x_{n}\right\}_{n=-1}^{\infty}$ consists of a single semi-cycle, then $\left\{x_{n}\right\}_{n=-1}^{\infty}$ converges to $\bar{x}=2 /(1-\beta)$;

(ii) if $\left\{x_{n}\right\}_{n=-1}^{\infty}$ consists of at least two semi-cycles, then $\left\{x_{n}\right\}_{n=-1}^{\infty}$ converges to a period-2 solution of (1).

Proof: It is known by Lemma 6 that if $\left\{x_{n}\right\}_{n=-1}^{\infty}$ consists of a single semi-cycle, then $\left\{x_{n}\right\}_{n=-1}^{\infty}$ converges to $\bar{x}$. Otherwise, by Lemma 7, $\left\{x_{n}\right\}_{n=-1}^{\infty}$ is oscillatory and, except possibly for the first semi-cycle, every semi-cycle has length 1 and every term of $\left\{x_{n}\right\}_{n=-1}^{\infty}$ is greater than $\alpha=1$. The proof of the second part follows from Corollary 1 and Lemma 1.

\section{Stability analysis}

Lemma 8 For the equilibrium point $\bar{x}=(1+\alpha) /(1-$ $\beta$ ) of (1), we have

(i) $\bar{x}$ is locally asymptotically stable if $\alpha>1$;

(ii) $\bar{x}$ is unstable if $0 \leqslant \alpha<1$.

Proof: The linearized equation of (1) about $\bar{x}$ is

$$
y_{n+1}=A y_{n}+B y_{n-1},
$$

where $A=-(1-\beta) /(1+\alpha)$ and $B=(1+\alpha \beta) /(1+\alpha)$. Let $0 \leqslant \beta<1$.

(i) If $\alpha>1$, then

$$
|A|+B-1=\frac{(1-\alpha)(1-\beta)}{1+\alpha}<0
$$

and

$$
1-B=\frac{\alpha(1-\beta)}{1+\alpha}<2,
$$

and hence, by Theorem 1(iii), $\bar{x}$ is locally asymptotically stable.

(ii) If $0 \leqslant \alpha<1$, then

$$
A^{2}+4 B>0
$$

and

$$
|A|-|1-B|=\frac{(1-\alpha)(1-\beta)}{1+\alpha}>0,
$$

and hence, by Theorem 1(iv), $\bar{x}$ is unstable.

Lemma 9 Let $\alpha>1$, and let $\left\{x_{n}\right\}_{n=-1}^{\infty}$ be a positive solution of (1). Then

$$
\begin{aligned}
\frac{\alpha}{1-\beta}+\frac{\alpha-1}{\alpha} & \leqslant \liminf _{n \rightarrow \infty} x_{n} \\
& \leqslant \limsup _{n \rightarrow \infty} x_{n} \leqslant \frac{\alpha^{2}}{(\alpha-1)(1-\beta)} .
\end{aligned}
$$

Proof: Because of Lemmas 6 and 7, it may be assumed that every semi-cycle of $\left\{x_{n}\right\}_{n=-1}^{\infty}$ has length 1 , that $\alpha /(1-\beta)<x_{n}$ for all $n \geqslant-1$, and that $\alpha /(1-\beta)<x_{0}<(1+\alpha) /(1-\beta)<x_{-1}$. Note that for $n \geqslant 0$,

$$
\begin{aligned}
x_{2 n+1} & =\alpha+\beta x_{2 n-1}+\frac{x_{2 n-1}}{x_{2 n}} \\
& <\alpha+\left(\beta+\frac{1-\beta}{\alpha}\right) x_{2 n-1} .
\end{aligned}
$$

Thus

$$
x_{2 n+1}<\alpha+\alpha\left(\beta+\frac{1-\beta}{\alpha}\right)+\left(\beta+\frac{1-\beta}{\alpha}\right)^{2} x_{2 n-3} .
$$

Successive application of the previous inequality leads to

$$
\begin{array}{r}
x_{2 n+1}<\frac{\alpha^{2}}{(\alpha-1)(1-\beta)}\left[1-\left(\beta+\frac{1-\beta}{\alpha}\right)^{n}\right] \\
+\left(\beta+\frac{1-\beta}{\alpha}\right)^{n} x_{-1} .
\end{array}
$$

Since $\beta+(1-\beta) / \alpha<1$, it follows from (10) with $m=0$ and (11) that

$$
\limsup _{n \rightarrow \infty} x_{n} \leqslant \frac{\alpha^{2}}{(\alpha-1)(1-\beta)} \text {. }
$$

That is, for any $\varepsilon>0$, there exists $N \geqslant 0$ such that

$$
x_{2 n+1}<\frac{\alpha^{2}+\varepsilon}{(\alpha-1)(1-\beta)} \text { for all } n \geqslant N .
$$

Thus for any $n>N$,

$$
\begin{aligned}
x_{2 n} & =\alpha+\beta x_{2 n-2}+\frac{x_{2 n-2}}{x_{2 n-1}} \\
& >\alpha+\left(\beta+\frac{(\alpha-1)(1-\beta)}{\alpha^{2}+\varepsilon}\right) \frac{\alpha}{1-\beta} \\
& =\frac{\alpha}{1-\beta}+\frac{\alpha(\alpha-1)}{\alpha^{2}+\varepsilon} .
\end{aligned}
$$

Since $\varepsilon$ is arbitrary, it follows that

$$
\liminf _{n \rightarrow \infty} x_{n} \geqslant \frac{\alpha}{1-\beta}+\frac{\alpha-1}{\alpha} .
$$

The following theorem, also given in Ref. 7, will be useful to obtain the global asymptotic stability condition of the fixed point $\bar{x}$ of (1).

Theorem 4 Let $f:(0, \infty) \times(0, \infty) \rightarrow(0, \infty)$ be a continuous function and consider the difference equation

$$
x_{n+1}=f\left(x_{n}, x_{n-1}\right), \quad n=0,1, \ldots,
$$

where $x_{-1}, x_{0} \in(0, \infty)$. Suppose $f$ satisfies the following conditions: 
(i) there exist positive numbers $a$ and $b$ with $a<b$ such that

$$
a \leqslant f(x, y) \leqslant b \quad \text { for all } x, y \in[a, b]
$$

(ii) $f(x, y)$ is non-increasing in $x \in[a, b]$ for each $y \in[a, b]$, and $f(x, y)$ is non-decreasing in $y \in$ $[a, b]$ for each $x \in[a, b]$;

(iii) (12) has no period-2 solutions in $[a, b]$.

Then there exists exactly one equilibrium point $\bar{x}$ of (12) which lies in $[a, b]$. Also, every solution of (12) which lies in $[a, b]$ converges to $\bar{x}$.

Theorem 5 Let $\alpha>1$. Then $\bar{x}=(1+\alpha) /(1-\beta)$ is a globally asymptotically stable equilibrium point of (1).

Proof: It is known from Lemma 8 that $\bar{x}=(1+$ $\alpha) /(1-\beta)$ is a locally asymptotically stable equilibrium point of (1). Let $\left\{x_{n}\right\}_{n=-1}^{\infty}$ be a positive solution of (1). It suffices to show that

$$
\lim _{n \rightarrow \infty} x_{n}=\frac{1+\alpha}{1-\beta} .
$$

For $x, y \in(0, \infty)$, set $f(x, y)=\alpha+\beta y+y / x, a=$ $\alpha /(1-\beta)$, and $b=\alpha^{2} /[(\alpha-1)(1-\beta)]$. Then,

$$
\begin{aligned}
f(a, b) & =\alpha+\frac{\beta \alpha^{2}}{(\alpha-1)(1-\beta)}+\frac{\alpha}{\alpha-1} \\
& =\frac{\alpha^{2}}{(\alpha-1)(1-\beta)}=b
\end{aligned}
$$

and

$$
f(b, a)=\alpha+\frac{\alpha \beta}{1-\beta}+\frac{\alpha-1}{\alpha}=\frac{\alpha}{1-\beta}+\frac{\alpha-1}{\alpha}>a .
$$

Hence

$$
a \leqslant f(x, y) \leqslant b \quad \text { for all } x, y \in[a, b] .
$$

By Lemma 9,

$$
\begin{aligned}
\frac{\alpha}{1-\beta}<\frac{\alpha}{1-\beta}+ & \frac{\alpha-1}{\alpha} \leqslant \liminf _{n \rightarrow \infty} x_{n} \\
& \leqslant \limsup _{n \rightarrow \infty} x_{n} \leqslant \frac{\alpha^{2}}{(\alpha-1)(1-\beta)}
\end{aligned}
$$

and, by Theorem 4,

$$
\lim _{n \rightarrow \infty} x_{n}=\frac{1+\alpha}{1-\beta} .
$$

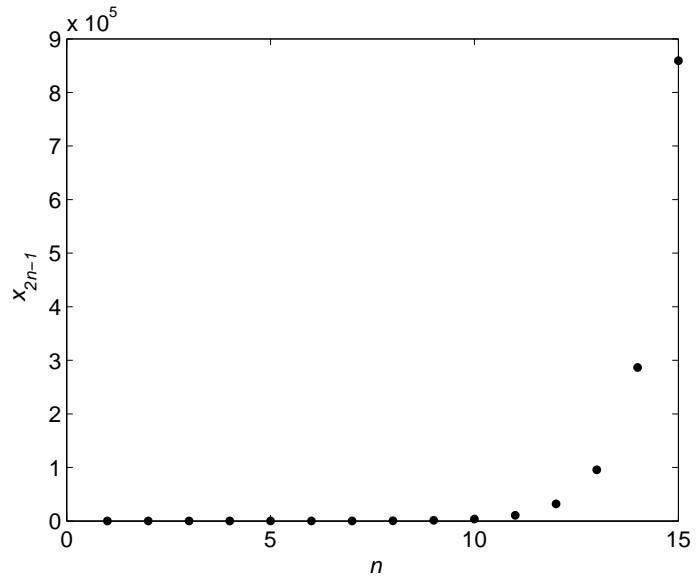

Fig. 1 Odd indexed terms of the solution of (13).

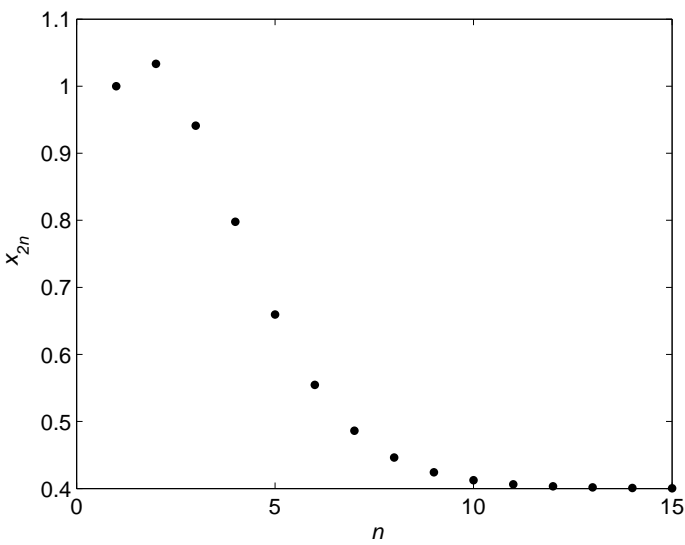

Fig. 2 Even indexed terms of the solution of (13).

\section{NUMERICAL EXAMPLES}

This part of the paper is devoted to some numerical tests to illustrate the theoretical results obtained in here.

Example 1 Consider the initial value problem (IVP)

$$
\begin{aligned}
x_{n+1} & =0.2+0.5 x_{n-1}+\frac{x_{n-1}}{x_{n}}, \quad n=0,1, \ldots, \\
x_{-1} & =1, \quad x_{0}=3 .
\end{aligned}
$$

Clearly, the conditions of Theorem 2 are satisfied and, as a result, $\lim _{n \rightarrow \infty} x_{2 n}=\infty$ and $\lim _{n \rightarrow \infty} x_{2 n+1}=\alpha /(1-\beta)=0.4$ as seen in Fig. 1 and Fig. 2.

Example 2 Consider the IVP

$$
\begin{aligned}
& x_{n+1}=1+0.5 x_{n-1}+\frac{x_{n-1}}{x_{n}}, \quad n=0,1, \ldots, \\
& x_{-1}=1, \quad x_{0}=5 .
\end{aligned}
$$




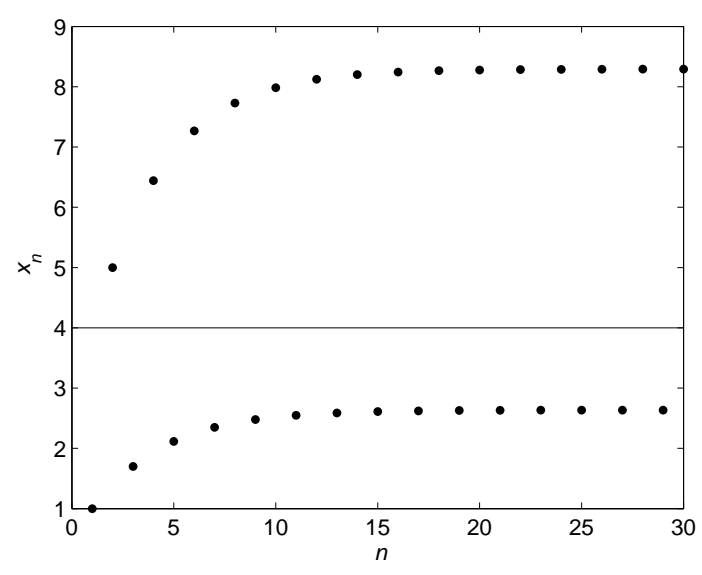

Fig. 3 The solution of (14).

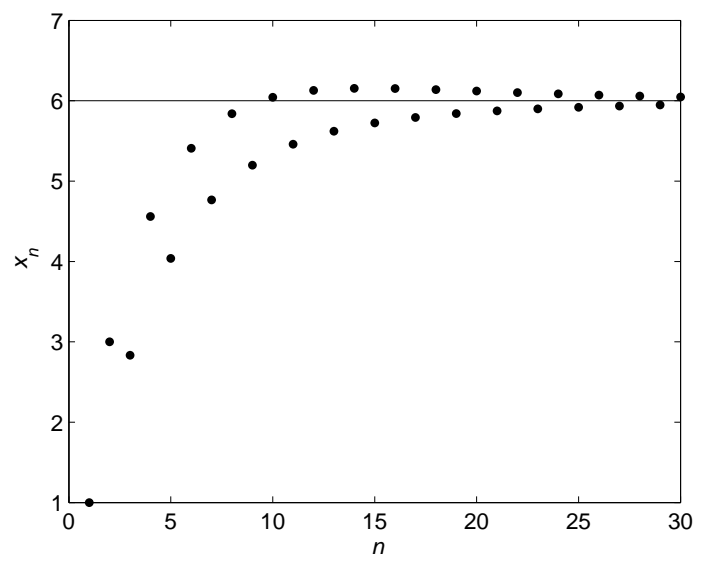

Fig. 4 The solution of (15).

Obviously, the solution $\left\{x_{n}\right\}_{n=-1}^{\infty}$ of (14) consists of at least two semi-cycles. Then, by Theorem 3, this solution converges to a period-2 solution as per Fig. 3.

Example 3 Consider the IVP

$$
\begin{aligned}
& x_{n+1}=2+0.5 x_{n-1}+\frac{x_{n-1}}{x_{n}}, \quad n=0,1, \ldots, \\
& x_{-1}=1, \quad x_{0}=3 .
\end{aligned}
$$

Since, in this example, $\alpha=2>1$, by Theorem 5 , the equilibrium point $\bar{x}=6$ of (15) is globally asymptotically stable. As it can be seen in Fig. 4, the solution $\left\{x_{n}\right\}$ of (15) converges to the fixed point $\bar{x}=6$.

Acknowledgements: The authors would like to express their sincere gratitude to Mr P. Danesh from the Atilim University Academic Writing and Advisory Centre for his help in the preparation of the manuscript, and to the anonymous referees for their time and effort in reviewing this work.

\section{REFERENCES}

1. Edelstein-Keshet L (2005) Mathematical Models in Biology, SIAM, Philadelphia.

2. Elaydi S (1999) An Introduction to Difference Equations, Springer-Verlag, New York.

3. Graef JR, Qian C, Spikes PW (1998) Stability in a population model. Appl Math Comput 89, 119-32.

4. Rosenkranz G (1983) On global stability of discrete population models. Math Biosci 64, 227-31.

5. Kelley WG, Peterson AC (2001) Difference Equations: An Introduction with Applications, Academic Press, New York.

6. Kocic VL, Ladas G (1993) Global Behavior of Nonlinear Difference Equations of Higher Order with Applications, Kluwer Academic Publishers, Dordrecht.

7. Kulenović MRS, Ladas G (2002) Dynamics of Second Order Rational Difference Equations with Open Problems and Conjectures, Chapman \& Hall/CRC, New York.

8. Stević $S$ (2007) On the recursive sequence $x_{n+1}=$ $A+\left(x_{n}^{p} / x_{n-1}^{r}\right)$. Discrete Dynam Nat Soc 2007, 40963.

9. Hamza AE (2006) On the difference equation $x_{n+1}=$ $\alpha+x_{n-1} / x_{n} . J$ Math Anal Appl 322, 668-74.

10. Stević S (2008) On the difference equation $x_{n+1}=$ $\alpha+\frac{x_{n-1}}{x_{n}}$. Comput Math Appl 56, 1159-71.

11. Amleh AM, Grove EA, Ladas G, Georgiou DA (1999) On the recursive sequence $x_{n+1}=\alpha+x_{n-1} / x_{n}$.J Math Anal Appl 233, 790-8.

12. DeVault R, Kent C, Kosmala W (2003) On the recursive sequence $x_{n+1}=p+\frac{x_{n-k}}{x_{n}}$. J Difference Equat Appl 9, 721-30.

13. He W-S, Li W-T, Yan X-X (2004) On the recursive sequence $x_{n+1}=\alpha+\left(x_{n-k} / x_{n}\right)$. Appl Math Comput 151, 879-85. 